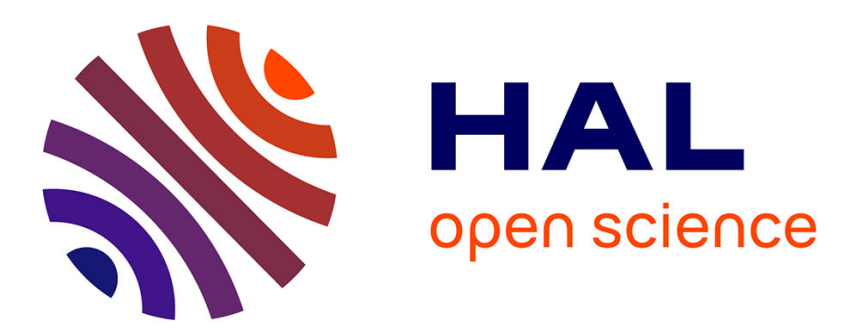

\title{
On the implementation of the continuum shell finite element SHB8PS and application to sheet forming simulation
}

Abdellah Salahouelhadj, Farid Abed-Meraim, Hocine Chalal, Tudor Balan

\section{To cite this version:}

Abdellah Salahouelhadj, Farid Abed-Meraim, Hocine Chalal, Tudor Balan. On the implementation of the continuum shell finite element SHB8PS and application to sheet forming simulation. American Institute of Physics Conf. Proc., 2011, 1353, pp.1203-1208. 10.1063/1.3589680 . hal-01206468

\section{HAL Id: hal-01206468 \\ https://hal.science/hal-01206468}

Submitted on 29 Sep 2015

HAL is a multi-disciplinary open access archive for the deposit and dissemination of scientific research documents, whether they are published or not. The documents may come from teaching and research institutions in France or abroad, or from public or private research centers.
L'archive ouverte pluridisciplinaire HAL, est destinée au dépôt et à la diffusion de documents scientifiques de niveau recherche, publiés ou non, émanant des établissements d'enseignement et de recherche français ou étrangers, des laboratoires publics ou privés. 


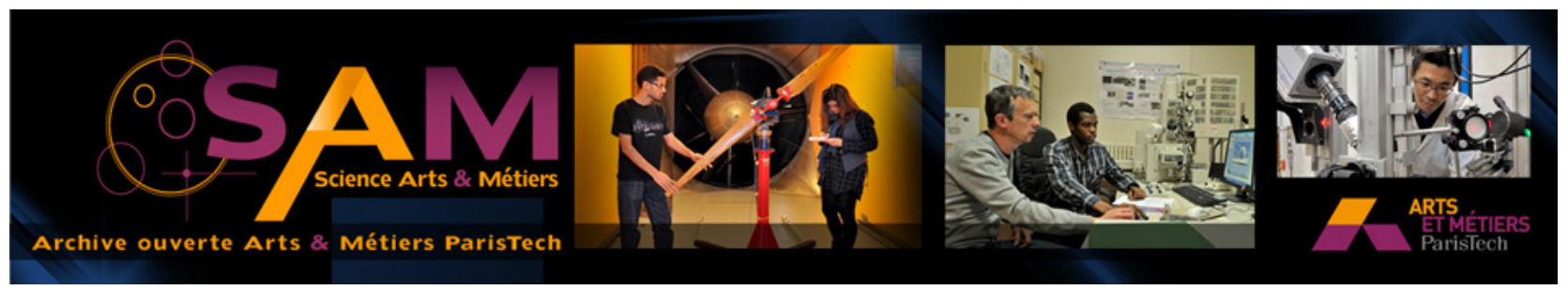

\section{Science Arts \& Métiers (SAM)}

is an open access repository that collects the work of Arts et Métiers ParisTech researchers and makes it freely available over the web where possible.

This is an author-deposited version published in: http://sam.ensam.eu

Handle ID: .http://hdl.handle.net/10985/10211

\section{To cite this version :}

Abdellah SALAHOUELHADJ, Farid ABED-MERAIM, Hocine CHALAL, Tudor BALAN - On the implementation of the continuum shell finite element SHB8PS and application to sheet forming simulation - American Institute of Physics Conf. Proc. - Vol. 1353, p.1203-1208 - 2011 


\title{
On the implementation of the continuum shell finite element SHB8PS and application to sheet forming simulation
}

\author{
A. Salahouelhadj, F. Abed-Meraim, H. Chalal and T. Balan \\ Laboratoire d'Etude des Microstructures et de Mécanique des Matériaux, LEM3 - UMR CNRS 7239 \\ Arts et Métiers ParisTech-Metz \\ 4 rue A. Fresnel, 57078 Metz Cedex 03, France
}

\begin{abstract}
In this contribution, the formulation of the SHB8PS continuum shell finite element is extended to anisotropic elastic-plastic behavior models with combined isotropic-kinematic hardening at large deformations. The resulting element is then implemented into the commercial implicit finite element code Abaqus/Standard via the UEL subroutine. The SHB8PS element is an eight-node, three-dimensional brick with displacements as the only degrees of freedom and a preferential direction called the thickness. A reduced integration scheme is adopted using an arbitrary number of integration points along the thickness direction and only one integration point in the other directions. The hourglass modes due to this reduced integration are controlled using a physical stabilization technique together with an assumed strain method for the elimination of locking. Therefore, the element can be used to model thin structures while providing an accurate description of the various through-thickness phenomena. Its performance is assessed through several applications involving different types of non-linearities: geometric, material and that induced by contact. Particular attention is given to springback prediction for a NUMISHEET benchmark problem.
\end{abstract}

Keywords: solid-shell element, reduced integration, physical stabilization, assumed strain method, elastic-plastic behavior, sheet metal forming, springback.

PACS: $02.70 . \mathrm{Dh}$

\section{INTRODUCTION}

During the last decade, considerable effort has been devoted to the development of eight-node solid-shell elements for modeling of thin structures (e.g. [1-4]). As they use linear interpolation for efficiency reasons, these elements exhibit various locking phenomena which need to be cured in order to preserve the desired accuracy. Nevertheless, compared to conventional shell elements they have many advantages: the use of full three-dimensional constitutive laws, direct calculation of thickness variations, easy treatment to update configurations (no rotational degrees of freedom used), and simple connection with three-dimensional elements since displacements are the only degrees of freedom. For sheet forming applications, key features like double-sided contact and increased accuracy with only one layer of elements through the thickness make these elements particularly attractive.

The reduced integration technique, initiated by the works of Zienkiewicz et al. [5] and Hughes et al. [6], was the first successful solution to alleviate some locking pathologies. Finite elements using this method are very efficient due to their low numerical cost. However, stabilization techniques are needed in order to control the spurious zeroenergy deformation modes (or hourglass modes) induced by this reduced integration.

In order to circumvent locking phenomena for three-dimensional low-order elements, several authors have used the enhanced assumed strain (EAS) method, based on Simo and Rifai's pioneer work [7]. The basis of such element formulations is given by the mixed variational principle in which the so-called incompatible strain and stress act as additional independent variables. Recent investigations have combined EAS and reduced integration techniques to derive efficient and accurate elements. As examples, some authors used a fixed number of Gauss points in the thickness direction [1-4].

The SHB8PS is one such element that has been recently developed [1, 2], based on in-plane one-point numerical quadrature with eight physical nodes and using an arbitrary number of integration points through the thickness direction. This avoids the use of several layers of elements in order to increase the number of integration points in 
the thickness, e.g. for metal forming problems. The hourglass modes caused by this reduced integration are efficiently controlled by a physical stabilization technique based on the assumed strain method [8].

In the current contribution, the formulation of the SHB8PS solid-shell finite element is extended to anisotropic elastic-plastic behavior models with combined isotropic-kinematic hardening at large deformations. The resulting element is then implemented into the commercial implicit finite element code Abaqus/Standard via the UEL subroutine. Its good performance is demonstrated through non-linear benchmark problems involving large strains, plasticity and contact. Particular attention is given to springback prediction for a NUMISHEET benchmark problem.

\section{FORMULATION OF THE SHB8PS ELEMENT}

\subsection{Finite element interpolation}

SHB8PS is an eight-node, isoparametric hexahedral element with linear interpolation. It has a set of $n_{\text {int }}$ integration points chosen along the thickness direction $\zeta$ in the local coordinate frame (see Fig.1).

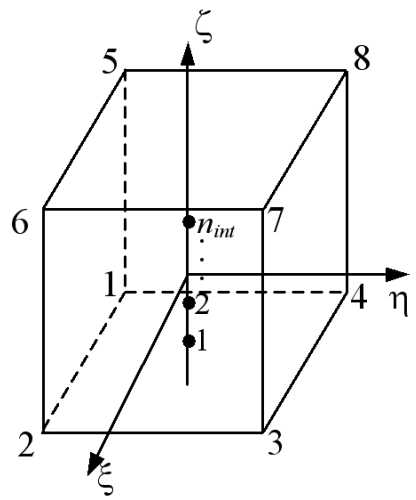

FIGURE 1. SHB8PS reference geometry.

The spatial coordinates $x_{i}$ and displacements $u_{i}$ of any point in the element are related to the nodal coordinates and nodal displacements $x_{i I}$ and $u_{i I}$, respectively, using the classic linear isoparametric shape functions $N_{I}$ :

$$
\begin{aligned}
& x_{i}=x_{i I} N_{I}(\xi, \eta, \zeta)=\sum_{I=1}^{8} x_{i I} N_{I}(\xi, \eta, \zeta) \\
& u_{i}=u_{i I} N_{I}(\xi, \eta, \zeta)=\sum_{I=1}^{8} u_{i I} N_{I}(\xi, \eta, \zeta)
\end{aligned}
$$

Subscript $i$ varies from 1 to 3 and represents the direction of the spatial coordinates. Subscript $I$ varies from 1 to 8 .

\subsection{Discretized gradient operator}

First, we introduce the $\underline{b}_{i}(i=1, \ldots, 3)$ vectors, representing the derivatives of the shape functions at the origin of the reference coordinate system, defined by Hallquist [9] as

$$
\underline{b}_{i}^{T}=\underline{N}_{, i}(0,0,0) \quad i=1,2,3
$$

The displacement gradient can then be written as follows (see Belytschko and Bindeman [8]):

$$
u_{i, j}=\left(\underline{b}_{j}^{T}+\sum_{\alpha=1}^{4} h_{\alpha, j} \underline{\gamma}_{\alpha}^{T}\right) \cdot \underline{d}_{i}=\left(\underline{b}_{j}^{T}+h_{\alpha, j} \underline{\gamma}_{\alpha}^{T}\right) \cdot \underline{d}_{i}
$$

where $\underline{d}_{i}$ are the nodal displacement vectors. The functions $h_{\alpha}$ and vectors $\underline{\gamma}_{\alpha}(\alpha=1, \ldots, 4)$ are given by

$$
h_{1}=\eta \zeta, h_{2}=\zeta \xi, h_{3}=\xi \eta, h_{4}=\xi \eta \zeta
$$




$$
\underline{\gamma}_{\alpha}=\frac{1}{8}\left[\underline{h}_{\alpha}-\sum_{j=1}^{3}\left(\underline{h}_{\alpha}^{T} \cdot \underline{x}_{j}\right) \underline{b}_{j}\right]
$$

The discretized gradient operator can be written as

$$
\underline{\underline{B}}=\left[\begin{array}{ccc}
\underline{b}_{x}^{T}+h_{\alpha, x} \underline{\gamma}_{\alpha}^{T} & \underline{0} & \underline{0} \\
\underline{0} & \underline{b}_{y}^{T}+h_{\alpha, y} \underline{\gamma}_{\alpha}^{T} & \underline{0} \\
\underline{0} & \underline{0} & \underline{b}_{z}^{T}+h_{\alpha, z} \underline{\gamma}_{\alpha}^{T} \\
\underline{b}_{y}^{T}+h_{\alpha, y} \underline{\gamma}_{\alpha}^{T} & \underline{b}_{x}^{T}+h_{\alpha, x} \underline{\gamma}_{\alpha}^{T} & \underline{0} \\
\underline{0} & \underline{b}_{z}^{T}+h_{\alpha, z} \underline{\gamma}_{\alpha}^{T} & \underline{b}_{y}^{T}+h_{\alpha, y} \underline{\gamma}_{\alpha}^{T} \\
\underline{b}_{z}^{T}+h_{\alpha, z} \underline{\gamma}_{\alpha}^{T} & \underline{0} & \underline{b}_{x}^{T}+h_{\alpha, x} \underline{\gamma}_{\alpha}^{T}
\end{array}\right]
$$

\subsection{Stabilization and assumed strain method}

The particular location of the integration points along a line generates six so-called hourglass modes. The control of the hourglass modes of the SHB8PS element is achieved by adding a stabilization component $\underline{K}_{S T A B}$ to the element stiffness matrix $\underline{K}_{e}$. This part is drawn from the work of Belytschko and Bindeman [8], who applied an efficient stabilization technique together with an assumed strain method. The stabilization forces are consistently derived in the same way. Moreover, the discretized gradient operator is projected onto an appropriate sub-space in order to eliminate shear and membrane locking.

In this approach, the $\underline{b}_{i}$ vectors (Eq. (3)) are replaced by the mean value of the derivatives of the shape functions over the element, denoted by $\hat{b}_{i}$, as proposed by Flanagan and Belytschko [10]:

$$
\underline{\hat{b}}_{i}^{T}=\frac{1}{\Omega_{e}} \int_{\Omega_{e}} \underline{N}_{, i}(\xi, \eta, \zeta) d \Omega, \quad i=1,2,3
$$

Then, vectors $\underline{\gamma}_{\alpha}$ are replaced by vectors $\underline{\hat{\gamma}}_{\alpha}$ where the $\underline{b}_{i}$ vectors are simply substituted by $\underline{\hat{b}}_{i}$. A modified discretized gradient operator $\underline{\underline{\hat{B}}}$ can be constructed in the same way. It can be shown that the terms of the $\underline{\underline{\hat{B}}}$ operator vanish for $\alpha=3,4$. In other words, the $\underline{\underline{\hat{B}}}$ operator reduces to its $\underline{\underline{\hat{B}}}_{12}$ part defined identically but where $\alpha$ varies only from 1 to 2 . Then, the remaining part $\underline{\underline{B}}_{34}$ of $\underline{\underline{\hat{B}}}$, which vanishes at the integration points, is further projected as $\underline{\underline{\hat{B}}}_{34}$. One can project the $\underline{\underline{\hat{B}}}$ operator onto a $\underline{\underline{\hat{B}}}$ operator as:

$$
\underline{\underline{\hat{B}}}=\underline{\underline{B}}_{12}+\underline{\underline{\hat{B}}}_{34}
$$

where $\underline{\underline{\hat{B}}}_{34}$ is given by:

$$
\underline{\hat{\hat{B}}}_{34}=\left[\begin{array}{ccc}
\sum_{\alpha=3}^{4} h_{\alpha, x} \hat{\gamma}_{\alpha}^{T} & \underline{0} & \underline{0} \\
\underline{0} & \sum_{\alpha=3}^{4} h_{\alpha, y} \underline{\hat{\gamma}}_{\alpha}^{T} & \underline{0} \\
\underline{0} & \underline{0} & h_{3, z} \hat{\gamma}_{3}^{T} \\
\underline{0} & \underline{0} & \underline{0} \\
\underline{0} & \underline{0} & \underline{0} \\
\underline{0} & \underline{0} & h_{4, x} \underline{\hat{\gamma}}_{4}^{T}
\end{array}\right]
$$


The stiffness matrix $\underline{\underline{K}}_{e}$ takes the form:

$$
\underline{\underline{K}}_{e}=\int_{\Omega_{e}} \underline{\underline{\hat{B}}}^{T} \cdot \underline{\underline{C}}^{e p} \cdot \underline{\underline{\hat{B}}} d \Omega+\underline{\underline{K}}_{\text {Geom }}=\underline{\underline{K}}_{12}+\underline{\underline{K}}_{S T A B}+\underline{\underline{K}}_{\text {Geom }}
$$

where the first term $\underline{\underline{K}}_{12}$ is evaluated at the integration points as

$$
\underline{K}_{12}=\int_{\Omega_{e}} \underline{\underline{\hat{B}}}_{12}^{T} \cdot \underline{\underline{C}}^{e p} \cdot \underline{\underline{B}}_{12} d \Omega=\sum_{I=1}^{n_{\text {int }}} \omega\left(\zeta_{I}\right) J\left(\zeta_{I}\right) \underline{\underline{\hat{B}}}_{12}^{T}\left(\zeta_{I}\right) \cdot \underline{\underline{C}}^{e p} \cdot \underline{\hat{B}}_{12}\left(\zeta_{I}\right)
$$

In this equation, $J\left(\zeta_{I}\right)$ is the Jacobian of the transformation between the reference and the current configurations; $\omega\left(\zeta_{I}\right)$ is the corresponding weight, while $\underline{\underline{C}}^{e p}=\frac{\partial \Delta \underline{\sigma}}{\partial \Delta \underline{\varepsilon}}$ is the elastic-plastic tangent modulus. The geometric stiffness matrix $\underline{\underline{K}}_{\text {Geom }}$ is due to the non-linear (quadratic) part of the strain tensor and $\underline{\underline{K}}_{\text {STAB }}$ represents the stabilization stiffness given by equation:

$$
\underline{\underline{K}}_{S T A B}=\int_{\Omega_{e}} \underline{\hat{\underline{B}}}_{12}^{T} \cdot \underline{\underline{C}}^{e p} \cdot \underline{\underline{\hat{B}}}_{34} d \Omega+\int_{\Omega_{e}} \underline{\hat{\underline{B}}}_{34}^{T} \cdot \underline{\underline{C}}^{e p} \cdot \underline{\underline{\hat{B}}}_{12} d \Omega+\int_{\Omega_{e}} \underline{\underline{\hat{B}}}_{34}^{T} \cdot \underline{\underline{C}}^{e p} \cdot \underline{\underline{\hat{B}}}_{34} d \Omega
$$

In a similar way, the internal forces of the element can be written as

$$
\underline{f}^{i n t}=\sum_{I=1}^{n_{\text {int }}} \omega\left(\zeta_{I}\right) J\left(\zeta_{I}\right) \underline{\hat{B}}_{12}^{T}\left(\zeta_{I}\right) \cdot \underline{\sigma}\left(\zeta_{I}\right)+\underline{f}^{S T A B}
$$

where $\underline{f}^{S T A B}$ represents the stabilization forces.

The stabilization terms are calculated in a co-rotational coordinate system [8].

\section{NUMERICAL EXAMPLE: UNCONSTRAINED CYLINDRICAL BENDING}

The example of the unconstrained cylindrical bending test proposed as springback benchmark in NUMISHEET 2002 is studied [11]. This application allows us to evaluate the performance of the SHB8PS element, implemented in Abaqus/Standard, in presence of geometric, material and contact non-linearities. This benchmark involves a bending-dominated deformation since there is no blank holder. The problem has complex contact boundary conditions during the forming process and the springback after forming is severe. The geometry of the problem is illustrated in Fig.2 and the geometric parameters are summarized in Table 1.

The material under investigation is a High Strength Steel, which is supposed elastic-plastic with isotropic hardening following Swift law:

$$
\sigma_{Y}=K\left(\varepsilon_{0}+\varepsilon_{e q}^{P}\right)^{n}
$$

where $K, \varepsilon_{0}$ and $n$ represent the material parameters and $\varepsilon_{e q}^{P}$ is the equivalent plastic strain. The Young modulus $\mathrm{E}=2.175 \times 10^{5} \mathrm{MPa}$ and the Poisson ration $v=0.3$. Further $K=645.24, n=0.25177$ and $\varepsilon_{0}=0.0102$. The friction coefficient of the interaction between surfaces punch-sheet and die-sheet is $\mu=0.14812$.

The amount of springback is quantified by the angle $\theta$ as defined in Fig.3. This angle is measured after forming at the maximum punch displacement and after springback. The tools are defined as analytical rigid surfaces.

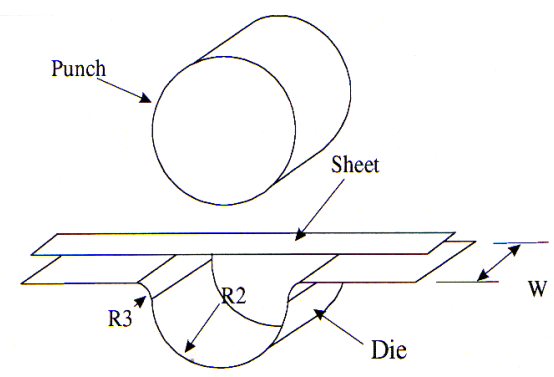

FIGURE 2. Tool geometry for the unconstrained bending problem 
TABLE 1. Geometric parameters of the unconstrained cylindrical bending problem

\begin{tabular}{lclc}
\hline Geometric parameter & {$[\mathbf{m m}]$} & Geometric parameter & {$[\mathbf{m m}]$} \\
\hline Punch radius & 23.5 & Length of the sheet & 120.0 \\
Die radius (R2) & 25.0 & Thickness of the sheet & 1.0 \\
Die shoulder (R3) & 4.0 & Width of the sheet & 30.0 \\
Width of tools (W) & 50.0 & Punch stroke & 28.5 \\
\hline
\end{tabular}

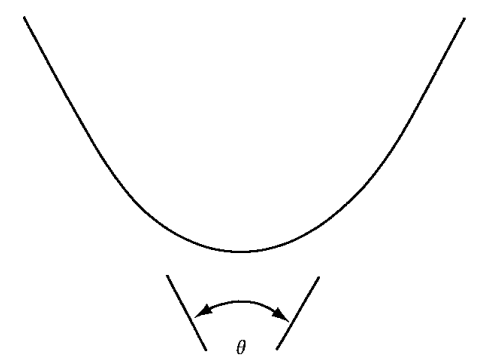

FIGURE 3. Definition of the angle to measure springback for the unconstrained cylindrical bending problem

The SHB8PS element is compared with both solid and shell elements. Indeed, it is well-known that in applications of sheet metal forming, shell elements have difficulties in dealing with double-sided contact - while conventional solid elements require several element layers to capture bending effects. In the present work, the simulations carried out with the SHB8PS element use only one element layer through the thickness. For symmetry reasons, only one quarter of the blank is discretized by means of 150 SHB8PS elements in the length and only one element over the width of the sheet. The analysis with the SHB8PS element is carried out using five Gauss points in the thickness direction because elastic-plastic applications require, in general five integration points in minimum to describe the strongly non-linear through-thickness stress distribution [2].

In order to validate the proposed solid-shell element, its predictions are compared to the experimental results of the NUMISHEET 2002 benchmarks. Two elements from the element library of the Abaqus code are also used in the comparison: the shell element S4R and the 3D continuum element C3D8I. Again, 150 uniformly distributed elements are used in the length direction for these two elements. However, two layers of C3D8I elements are required in the thickness direction in order to represent the stress distribution due to bending with sufficient accuracy. Also, ten C3D8I elements are used along the width direction in order to keep their aspect ratio in acceptable limits. Fig. 4 displays the punch force versus punch displacement curves predicted by the three elements, along with the experimental results (BE-1,.., BE-4) from Meinders et al. [11].

Fig.4 shows that the numerical results obtained with SHB8PS element are the closest to the experimental results and they lay close to the solid element predictions. The slight differences between the two may be due to the different number and distribution of integration points along the thickness direction. The S4R element has too soft behavior with respect to SHB8PS and C3D8I elements.

The springback angles are also investigated, as they were also experimentally measured [11]. The springback phenomenon is particularly exacerbated in this unconstrained bending application, as illustrated in Fig.5. Table 2 summarizes the opening angles before and after springback for elements SHB8PS, C3D8I and S4R, compared to experiments. The simulated values with SHB8PS and C3D8I elements are close to each other and the closest to experiments. Comparing the numerical results to the experimental ones, the good performance of the SHB8PS solidshell element is confirmed.

TABLE 2. Measured and simulated opening angles before and after springback.

\begin{tabular}{c|cccc|ccc}
\hline \multirow{2}{*}{} & \multicolumn{4}{c}{ Experimental } & \multicolumn{3}{c}{ Simulated } \\
\cline { 2 - 8 } & BE-01 & BE-02 & BE-03 & BE-04 & SHB8PS & C3D8I & S4R \\
\hline forming & 22.7707 & 22.0064 & 23.0255 & 20.8599 & 23.0692 & 22.5820 & 33.3078 \\
\hline Springback & 37.4212 & 35.6787 & 30.9036 & 35.3636 & 36.3952 & 32.0832 & 43.9071 \\
\hline
\end{tabular}




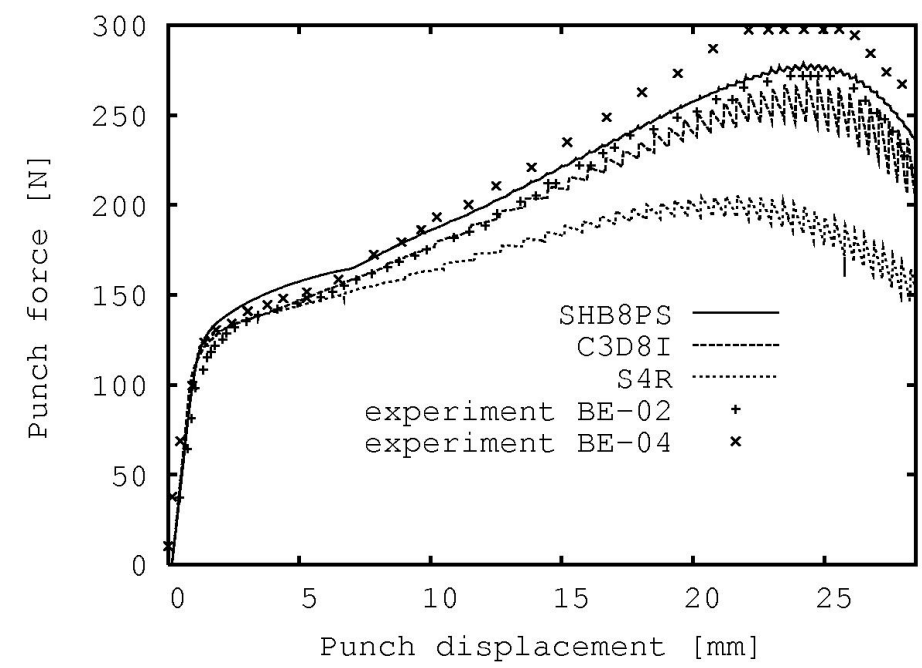

FIGURE 4. Punch force vs. punch displacement plots for High Strength Steel

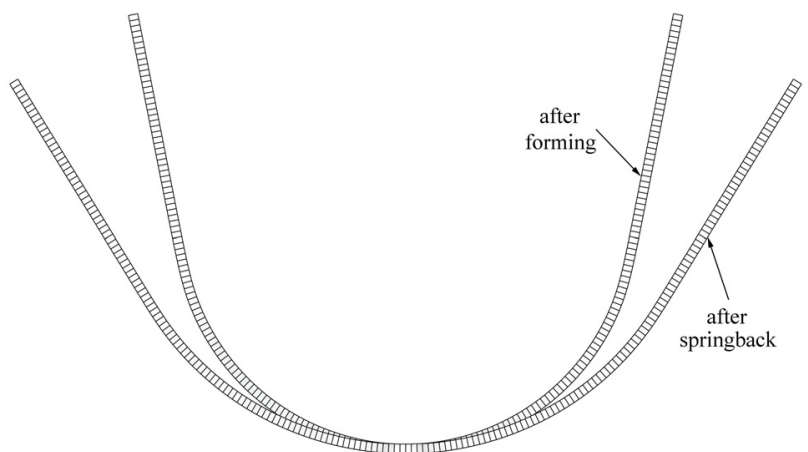

FIGURE 5. Deformed shape of the sheet in the unconstrained bending problem.

\section{ACKNOWLEDGMENTS}

The authors are grateful to the Agence Nationale de la Recherche-ANR (France) for its financial support through the Mat\&Pro project FORMEF. The first author is grateful to the Region Lorraine for its financial support.

\section{REFERENCES}

1. F. Abed-Meraim and A. Combescure, International Journal for Numerical Methods in Engineering, 80, 1640-1686 (2009).

2. A. Legay and A. Combescure, International Journal for Numerical Methods in Engineering, 57, 1299-1322 (2003).

3. R.J. Alves de Sousa, R.P.R. Cardoso, R.A. Fontes Valente, J.W. Yoon, J.J. Grácio and R.M. Natal Jorge, International Journal for Numerical Methods in Engineering, 67, 160-188 (2006).

4. S. Reese, Computer Methods in Applied Mechanics and Engineering, 194, 4685-4715 (2005).

5. O. Zienkiewicz, R. Taylor and J. Too, International Journal for Numerical Methods in Engineering, 3, 275-290 (1971).

6. T. Hughes, M. Cohen and M. Haroun, Nuclear Engineering Design, 46, 203-222 (1978).

7. J. Simo and M. Rifai, International Journal for Numerical Methods in Engineering, 29, 1595-1638 (1990).

8. T. Belytschko and L. Bindeman, Computer Methods in Applied Mechanics and Engineering, 105, 225-260 (1993).

9. J. Hallquist, "Theoretical manual for DYNA3D". Tech. Rep. Report UC1D-19401, Lawrence Livermore National Laboratory, Livermore, CA, 1983.

10. D. Flanagan and T. Belytschko, International Journal for Numerical Methods in Engineering, 17, 679-706 (1981).

11. T. Meinders, A. Konter, S. Meijers, E. Atzema and H. Kappert, "A sensitivity analysis on the springback behavior of the unconstrained bending problem. In:NUMISHEET 2005, 6th international conference and workshop on numerical simulation of $3 D$ sheet metal forming processes. Detroit, Michigan, USA, 2005. 